hydrogen isotope deuterium as a function of the momentum of the quark that is struck by the electron. If there were no nucleon modification, this ratio would have a constant value of 1 . The fact that this ratio decreases as a function of momentum for a given nucleus indicates that individual nucleons in the nucleus are somehow modified. Moreover, the fact that this decrease occurs more rapidly if the mass of the nucleus is increased suggests that the EMC effect is enhanced for heavier nuclei.

The CLAS Collaboration has used electron-scattering data taken at Jefferson Lab to establish a relationship between the size of the EMC effect and the number of neutronproton SRC pairs in a given nucleus. A key feature of the work is the extraction of a mathematical function that includes the effect of SRC pairs on the scattering cross-section and that is shown to be independent of the nucleus. This universality provides strong confirmation of the correlation between the EMC effect and neutron-proton SRC pairs. The results indicate that the nucleon modification is a dynamical effect that arises from local density variations, as opposed to being a static, bulk property of the medium in which all nucleons are modified by the average nuclear field.

The authors have focused on neutronproton SRC pairs for a particular reason: it turns out that these pairs are more common than their neutron-neutron or proton-proton

counterparts. In this sense, the nucleons are isophobic; that is, similar nucleons are less likely to pair up than are dissimilar nucleons. Therefore, owing to the asymmetry in the numbers of neutrons and protons in medium-mass and heavy nuclei, the probability of protons forming neutron-proton SRC pairs increases roughly as the ratio of neutrons to protons, whereas the probability of neutrons doing this tends to plateau ${ }^{10}$. The CLAS Collaboration

"A lingering question had been whether protons and neutrons were modified when inside a nucleus." has used this specific feature to solidify its conclusions by demonstrating a clear difference between the per-proton and per-neutron EMC effects for asymmetric nuclei heavier than carbon. The fact that this distinction emerges directly from the data provides further support for the authors' interpretation that the nucleon modification arises from the formation of SRC pairs.

One implication of the present study is that information deduced about free neutrons from DIS experiments on deuterium or heavier nuclei needs to be corrected for the EMC effect to account for the modification of the neutrons in the nuclear medium. Another consequence concerns current and future experiments in which neutrinos or their antiparticles (antineutrinos) are scattered from asymmetric nuclei. Because protons and neutrons have different quark compositions, and because protons are more strongly affected by the in-medium modification than are neutrons, neutrino and antineutrino scattering cross-sections can show variations that could erroneously be attributed to an effect of some exotic physics - such as deficiencies in the standard model of particle physics, or possible mechanisms for understanding the asymmetry between matter and antimatter in the Universe. Before any such claim can be made, the differences in the EMC effect for protons and neutrons would have to be taken into account.

Gerald Feldman is in the Department of Physics, George Washington University, Washington DC 20052, USA.

e-mail:feldman@gwu.edu

1. Aubert, J. J. et al. Phys. Lett. B 123, 275-278 (1983).

2. The CLAS Collaboration. Nature $566,354-358$ (2019).

3. Rutherford, E. Phil. Mag. 21, 669-688 (1911)

4. Bloom, E. D. et al. Phys. Rev. Lett. 23, 930-934 (1969).

5. Breidenbach, M. et al. Phys. Rev. Lett. 23, 935-939 (1969).

6. Arnold, R. G. et al. Phys. Rev. Lett. 52, 727-730 (1984)

7. Gomez, J. et al. Phys. Rev. D 49, 4348-4372 (1994)

8. Seely, J. et al. Phys. Rev. Lett. 103, 202301 (2009).

9. Lubkin, G. B. Phys. Today 44, 17-20 (1991).

10.The CLAS Collaboration. Nature 560, 617-621 (2018).

\title{
Tumours use a metabolic twist to make lipids
}

\begin{abstract}
To survive and divide, cancer cells need a constant supply of lipid molecules called monounsaturated fatty acids. Tumours can achieve this by an unsuspected route that harnesses a metabolic pathway also used in hair follicles. SEE LETTER P.403
\end{abstract}

\section{MARTEINN THOR SNAEBJORNSSON \& ALMUT SCHULZE}

A bnormal cellular metabolism is a hallmark of cancer cells, from alterations in the pathways that use glucose to aberrant activation of lipid metabolism. Lipids are a highly complex class of molecule with many cellular functions ${ }^{1}$, one of the most important of which is to provide the building blocks for the synthesis of cellular lipid membranes. Most tissues in the adult body rely on lipids obtained from the diet or those made in the liver, but many cancer cells instead activate lipid-synthesis pathways to support their rapid proliferation ${ }^{2}$. This difference between normal and cancerous cells suggests a possible tumour-cell vulnerability that might be exploited therapeutically. Indeed, preventing the synthesis or modification of fatty acids (the building blocks for lipids) can reduce tumour growth in several animal models of cancer ${ }^{2}$, although this approach has not been successful in the clinic yet. Vriens et al. ${ }^{3}$ report results on page 403 that might indicate a way forward.

One reason that anticancer strategies targeting lipid metabolism have been ineffective in the clinic could be that alternative pathways compensate for the pathway that is blocked by a given drug. Vriens and colleagues have identified one such compensatory pathway in cancer cells that enables the cells to make monounsaturated fatty acids if the pathway that they normally use is blocked. This alternative pathway is known to act in oil-producing sebaceous glands in human hair follicles, and the authors' discovery has revealed that cancer cells can also harness this pathway to meet their metabolic demands.

The enzyme stearoyl-CoA desaturase (SCD) catalyses the formation of a specific double bond in palmitate, a saturated fatty acid (a fatty acid without a double bond), and this type of desaturation reaction is needed in the pathway that generates the monounsaturated fatty acids palmitoleate and oleate. These fatty acids are key building blocks for the phospholipids that are components of cellular membranes. The authors investigated the effect of an SCD inhibitor on human cancer cells grown in vitro. They found that some of the types of cancer cell tested were highly sensitive to SCD inhibition, and either stopped dividing or died, whereas others were insensitive and continued to divide. This was unexpected, because the predicted outcome of SCD inhibition would be the accumulation of saturated fatty acids that are toxic to cells at high concentrations ${ }^{4}$.

Vriens et al. found that cancer cells that are insensitive to SCD inhibition contain high levels of sapienate, a type of a monounsaturated fatty acid that is usually produced in the sebaceous gland. Sapienate is produced ${ }^{5}$ from palmitate by an enzyme called FADS2 (Fig. 1). FADS2 is also required ${ }^{6}$ in mammalian tissues for the processing of omega- 3 and omega- 6 essential fatty acids, which are those obtained from the diet.

The authors report that, relative to its expression in normal tissue, FADS2 expression is elevated in samples from human liver 


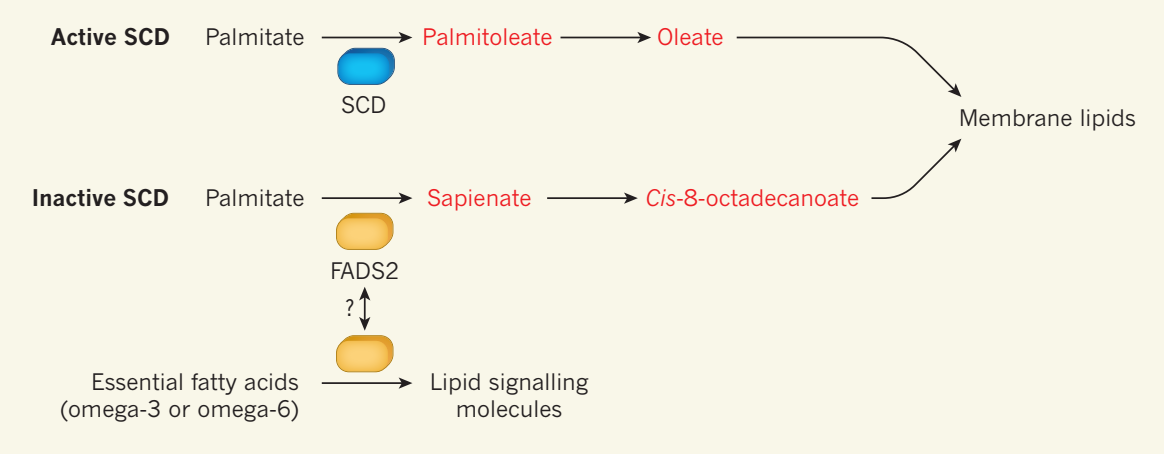

Figure 1 | Lipid-generating pathways in cancer cells. Vriens et al. ${ }^{3}$ used mouse models and different types of human cancer cell grown in vitro to investigate lipid production in tumours. The enzyme stearoyl-CoA desaturase (SCD) uses the molecule palmitate (a saturated fatty acid) to generate a monounsaturated fatty acid (examples of these are shown in red) called palmitoleate, which is used to produce oleate. Palmitoleate and oleate are building blocks for the generation of membrane lipids that are essential for cancer-cell survival. When SCD was inhibited in cancer cells, some cells still had the capacity to use palmitate to make monounsaturated fatty acids. These cells generated sapienate and cis-8-octadecanoate using the enzyme FADS2, which is mainly known for processing the essential fatty acids (those obtained from the diet) omega- 3 and omega- 6 fatty acids, to make lipid signalling molecules. FADS2 generates sapienate in normal cells of the oil-producing sebaceous gland in hair follicles ${ }^{5}$, and Vriens and colleagues' work reveals a previously unappreciated flexibility of cancer cells that allows them to use this enzyme to generate membrane lipids. This raises the question of whether switching to sapienate production when SCD is inhibited alters the cancer cells' ability to use FADS2 to make the type of lipid signalling molecules ${ }^{7}$ that help cells to evade targeting by the immune system.

and lung tumours. They note that sapienate is detectable in tumours from mouse models of liver cancer, and that, in these tumours, FADS2 expression correlates with resistance of the cancer cells to SCD inhibition. Interestingly, in an analogous manner to how oleate is formed from the elongation of palmitoleate, the monounsaturated fatty acid cis-8-octadecanoate is formed from the elongation of sapienate. The authors found that both sapienate and cis-8-octadecanoate are incorporated into the membrane lipids of cancer cells.

Vriens and colleagues next investigated whether the FADS2-dependent pathway for the synthesis of monounsaturated fatty acids could compensate for the lack of these compounds that usually occurs when SCD is inhibited. They indeed found that either engineering human cancer cells to express FADS2 or adding sapienate to cells enabled the survival of cancer cells grown in vitro that would usually die if SCD was inhibited. However, human cancer cells grown in vitro that were insensitive to SCD inhibition were killed by a combination of SCD inhibition and depletion of FADS2. In a mouse model of liver cancer that the authors tested, inhibition of both SCD and FADS2 caused a moderate reduction in tumour growth compared with tumour growth in animals in which neither enzyme was inhibited.

Experiments using human cells grown in vitro indicated that the activities of SCD and FADS2 are interdependent. The production of sapienate by FADS2 increased if SCD was inhibited. Conversely, when FADS2 activity was blocked, the synthesis of palmitoleate by SCD was enhanced. This flexibility in lipidproduction pathways is highly beneficial for rapidly dividing cancer cells that require a constant supply of monounsaturated fatty acids. However, the authors observed that depletion of FADS2 in the absence of SCD inhibition increased the proliferation of cancer cells, indicating that, although FADS2 might offer a way of generating monounsaturated fatty acids, it comes at a cost in terms of the cells' proliferative ability.

Accumulation of palmitate can shift the activity of FADS2 towards favouring palmitate as its substrate, and can thereby promote sapienate production ${ }^{7}$. This could therefore provide a fail-safe mechanism for producing monounsaturated fatty acids when SCD is blocked. Indeed, cis-8-octadecanoate was undetectable in samples of phospholipids from cancer cells in the absence of SCD inhibition, suggesting that no more than a low level of sapienate is generated in cells in which SCD is active.

Vriens and colleagues' work raises a number of questions. For example, which mechanisms control the level of expression of FADS2 in cancer cells? Considering that FADS2dependent production of sapienate is relevant only in the absence of SCD, it seems unlikely that sapienate production is the reason for high FADS2 expression in human cancer. It is probable instead that the main function of FADS2 in such cells is to perform its usual role in processing omega- 3 and omega- 6 fatty acids to generate lipid-signalling molecules involved in functions such as immune evasion ${ }^{8}$. Switching to sapienate production when SCD is inhibited might prevent FADS2 from performing its usual role and block the production of these signalling molecules.

It is not known whether the monounsaturated fatty acids produced by FADS2 functionally replace those produced by SCD. Incorporation of sapienate and cis-8-octadecanoate into membrane lipids could result in differences in membrane fluidity, curvature or the association of membrane proteins, compared with the corresponding characteristics of membrane lipids made with palmitoleate and oleate.

Another question arising from this study is whether the tumour microenvironment influences the dependence of cancer cells on SCD and FADS2. Cells can also obtain monounsaturated fatty acids through the uptake of a type of phospholipid called a lysophospholipid ${ }^{9}$. Hence, the levels of such molecules in the tumour microenvironment might determine whether inhibiting both SCD and FADS2 would be an effective way of killing cancer cells. Vriens et al. found that human liver cancer cells implanted in the livers of mice treated with an SCD inhibitor take up sapienate from the tumour microenvironment. This suggests that sapienate synthesis by FADS2 in the tumour is insufficient to satisfy its need for monounsaturated fatty acids. Moreover, consistent with this possibility, the inhibition of tumour growth observed after combined depletion of SCD and FADS2 in mice was only moderate. Perhaps inhibiting fatty acid uptake from the tumour microenvironment might help to block tumour growth when SCD and FADS2 are inhibited.

Vriens et al. provide a thought-provoking example of how cancer cells evolve to meet their metabolic needs. Tackling the complexity of the mechanisms involved remains a challenge for effectively targeting lipid metabolism in cancer therapy.

\section{Marteinn Thor Snaebjornsson and}

Almut Schulze are in the Department of Biochemistry and Molecular Biology,

Theodor Boveri Institute, Biocenter, University of Würzburg, Würzburg 97074, Germany. e-mails: marteinn.snaebjoernsson@uniwuerzburg.de;

almut.schulze@uni-wuerzburg.de

1. Shevchenko, A. \& Simons, K. Nature Rev. Mol. Cell Biol. 11, 593-598 (2010).

2. Röhrig, F. \& Schulze, A. Nature Rev. Cancer 16, 732-749 (2016)

3. Vriens, K. et al. Nature 566, 403-406 (2019).

4. Ackerman, D. \& Simon, M. C. Trends Cell Biol. 24, 472-478 (2014).

5. Ge, L., Gordon, J. S., Hsuan, C., Stenn, K. \& Prouty, S. M. J. Invest. Dermatol. 120, 707-714 (2003).

6. Zhang, J. Y., Kothapalli, K. S. D. \& Brenna, J. T. Curr. Opin. Clin. Nutr. Metab. Care 19, 103-110 (2016).

7. Park, H. G. et al. Biochim. Biophys. Acta 1861, 91-97 (2016).

8. Zelenay, S. et al. Cell 162, 1257-1270 (2015).

9. Kamphorst, J. J. et al. Proc. Natl Acad. Sci. USA 110, 8882-8887 (2013)

This article was published online on 6 February 2019. 